\title{
La ciudad de los buses de colores: empresas de transporte público, planes de desarrollo y crecimiento urbano en Cali, 1969-1993
}

\section{City of colored buses: Public transport companies, development plans and urban growth in Cali, 1969-1993}

\author{
Diana Vinasco Martínez \\ Universidad Icesi, Cali, Colombia \\ diana.vinasco@correounivalle.edu.co; dyvinasco@icesi.edu.co \\ http://orcid.org/0000-0002-0580-1160
}

Fecha de recepción: 30 de marzo del 2018 Fecha de aceptación: 22 de mayo del 2018

Disponible en línea: 27 de junio del 2018

\begin{abstract}
Sugerencia de citación: Vinasco Martínez, D. (2018). La ciudad de los buses de colores: empresas de transporte público, planes de desarrollo y crecimiento urbano en Cali, 1969-1993. tiempo\&economía, 5(2), 155-177, doi: http://dx.doi.org/10.21789/24222704.1344
\end{abstract}

\section{RESUMEN}

El presente artículo busca tejer un relato sobre la historia urbana de la ciudad de Cali entre las décadas del setenta y el noventa del siglo XX, a partir de la relación entre la operación de las empresas de transporte público, los planes de desarrollo y la expansión de la ciudad. Se analizará de qué manera los gobiernos locales y nacionales buscaron la organización del transporte y la planificación de la ciudad, pero sus estrategias fueron sobrepasadas por las realidades de la migración, el crecimiento urbano y las necesidades básicas de sus habitantes. Una de esas necesidades fue la movilidad, que fue cubierta, a su manera, por las empresas de transporte que constituyeron la alternativa de los sectores periféricos para acceder a la ciudad formal. 
Palabras clave: Transporte público, planes de desarrollo, crecimiento urbano, historia económica

Códigos JEL: R41, R28, R23

\section{ABSTRACT}

This paper aims to tell a story about the urban history of the city of Cali, Colombia, between the 1970's and 1990's from the perspective of the urban transport companies, the development plans undertaken at the time and the city expansion. It will analyze show local and national governments attempted to organize the transportation system and plan the city but their strategies were undermined by factors such as migration, urban growth and the basic needs of the urban inhabitants. One of those needs was mobility which was covered, in its own way, by the transport companies that came about as an alternative method for inhabitants of the outskirts to access the sectors of the city center.

Keywords: Public transport, development plans, urban growth, economic history

JEL Codes: R41, R28, R23 


\section{Introducción}

Un bus Azul Platiado(sic) pasó la esquina a toda y yo me paré y le pegué un berrido. Que esperara por mí ahora que decidí partir. El chofer voltió en la marcha y me miró. Pero no podía parar, ya había cruzado el semáforo, más bien hundió el acelerador para darme tiempo a que yo corriera hasta el paradero. Yo corrí, con estos pulmones que no funcionan nada. Ese es el bus que me sirve, el Azul Platiado pasa por la galería, todos los choferes son hombres violentos: le tengo piedra a los choferes.

Andrés Caicedo, Noche sin fortuna, 1976

Las problemáticas urbanas y de movilidad que enfrentan las ciudades colombianas en la actualidad, plantean la necesidad de una reflexión sobre la evolución del sector del transporte público en el país, y la forma en que las relaciones entre los gobiernos centrales y locales han contribuido a la persistencia o superación de dichas problemáticas. Estas no están relacionadas únicamente con las deficiencias en el transporte, sino también con la imposibilidad de construir proyectos de ciudad en los cuales la planeación no signifique simplemente, como señalaba Jacques Aprile-Gniset, corregir lo ya ejecutado.

La organización del transporte público urbano fue uno de los mayores retos que enfrentó la ciudad de Cali a lo largo del siglo xx. A pesar de ello, ha sido uno de los temas menos estudiados por las ciencias sociales y, en consecuencia, existen pocos trabajos que den cuenta del impacto que el transporte público -en modalidad de buses- ha tenido en el desarrollo histórico de la ciudad. Este artículo forma parte de un esfuerzo por contribuir a llenar este vacío, surge de un propósito por analizar la incidencia del transporte público en el desarrollo, la planificación y la formación de la ciudad, y a su vez, la incidencia que tuvo el desarrollo urbano de la ciudad en el modelo de transporte que se implementó en el siglo xx. Por ello, el análisis gira en torno a la pregunta por la importancia del transporte público para las políticas de planificación de la Ciudad y su configuración urbana.

Se analizan las décadas del setenta al noventa del siglo xx en Cali, periodo que puede ser definido como de crecimiento acelerado de las empresas de transporte y crisis del modelo de movilidad. Las nuevas políticas de planeación llevaron a la búsqueda de soluciones definitivas al transporte público y a "sobrediagnosticar" sus problemáticas, aunque sin llevar cabo soluciones reales. Asimismo, se hizo evidente el peso que tenían las empresas del transporte en la economía y la expansión de la ciudad. Cabe aclarar que, al hablar de transporte público se hace referencia exclusivamente a las empresas de buses urbanos que, aunque prestaban el servicio público de transporte y eran reguladas por entidades estatales, pertenecían a empresarios privados.

Para analizar estas cuestiones, se utilizará un enfoque de historia social, política e institucional, que asocia los conceptos de tiempo, espacio y sociedad, para argumentar que son los actores sociales los que modifican el espacio urbano de acuerdo con sus necesidades (Vásquez, 1980, p. 30). En el mismo sentido, señala Aprile-Gniset (1992, p. 751), "nacida de la formación social, la formación espacial es cambiante y se va modificando con ella y al ritmo impuesto por ella". Con ello, se busca construir un modelo de análisis que logre evidenciar la relación indisociable que existe entre la expansión urbana, las políticas de planeación y el transporte público en la ciudad de Cali. 
El documento inicia con una breve descripción de los planes de desarrollo formulados en Cali a partir de 1970, cuyas propuestas no lograron mayor impacto en las formas de urbanización de la ciudad, la cual creció con un gran porcentaje de informalidad y en relación estrecha con el desarrollo del transporte público. Luego se analizan las propuestas planteadas para el mejoramiento del transporte y los diagnósticos sobre la forma en que las empresas de transporte operaban; documentos que, siendo más analíticos que propositivos, no tuvieron mayor impacto en la organización de este sector, que funcionó bajo lógicas de lucro y en detrimento de la calidad del servicio, pero que, paradójicamente, contribuyó a la consolidación de los barrios informales y su conexión con el resto de la ciudad. El análisis histórico pretende evidenciar que la desconexión entre el gobierno nacional y local, respecto a la regulación y organización de este sector económico, retardó la adopción de soluciones reales y de largo plazo que contribuyeran a un mejor desempeño del transporte como factor de mejoramiento de la calidad de vida de los ciudadanos.

\section{Cali: planeación urbana vs. expansión incontrolada}

La ciudad de Cali, ubicada en el suroccidente colombiano, es la capital del departamento del Valle del Cauca y la tercera ciudad más poblada del país. Además de su ubicación estratégica como punto de conexión entre el centro del país y la costa del Pacífico, se ha convertido en el centro económico y político de la región y el principal centro urbano receptor de migraciones en el occidente del país.

Desde la década de 1940, la ciudad venía presentando altas cifras de crecimiento poblacional. Las migraciones sumaban alrededor de cincuenta mil habitantes al año. Para 1973, la ciudad alcanzó 991.549 habitantes, en 1985 contaba con 1.429 .026 y en 1993 ya alcanzaba 1.847.176, presionando la demanda de vivienda.

Según las cifras expuestas por Mosquera (1984), la demanda requería construir anualmente alrededor de 12.000 viviendas; sin embargo, entre 1973 y 1978 solo se construyeron aproximadamente 26.000, incluyendo las informales que representaban un 33\% del total. Hacia 1978 la ciudad ya requería 80.893 nuevas viviendas y en 1985 la cifra aumentó a 156.662. Por ello, la urbanización clandestina fue la principal forma de adquisición de vivienda para sectores populares, construcciones realizadas mediante sistemas de autoconstrucción y autofinanciación.

El auge de los barrios informales llevó a Cali a ser conocida como "la ciudad de las invasiones" (Mosquera, 1984, p. 10) y a aumentar otras problemáticas relacionadas con este fenómeno, pues los barrios informales requerían el suministro de servicios básicos como acueducto, energía, vías y transporte; servicios que empezaron a ser suplidos de manera precaria por los mismos habitantes en los barrios informales, y en muchos de los formales que habían sido entregados por programas de vivienda estatal pero sin servicios básicos. El agua se adquiría a través de pilas públicas y la energía por medio de conexiones realizadas por los mismos habitantes; asimismo, la precariedad de las vías dificultaba la comunicación de los habitantes de los barrios informales y periféricos con la ciudad formal. Sin embargo, dichos sectores, a pesar del problema que representaban para la ciudad, eran también su principal fuente de mano de obra, por lo cual era necesario garantizar su movilidad hacia el centro de la ciudad y las zonas industriales. 
Esta necesidad se hace evidente en el Decreto 492 de 1964: Por medio del cual se establece un estatuto municipal sobre el sistema de asignación, prolongación o recortes de las rutas urbanas de buses que funcionan en la ciudad de Cali. A través de este decreto la Alcaldía aprobó recorridos que atravesaban barrios aún no legalizados y los conectaban con la ciudad formal. El decreto no creo nuevas rutas, sino que legisló sobre recorridos y empresas que venían funcionando desde la década de 1940 (Vinasco, 2017, p. 49).

El documento evidencia que la mayoría de empresas de transporte ubicaron los sitios de salida de los buses - denominados controles de despacho- en barrios surgidos de manera informal o de reciente creación, muchos aún sin legalizar; lo que muestra que, así como los habitantes de los nuevos barrios en las periferias dependían para su conexión con la ciudad de las empresas de transporte, la creación y consolidación de las empresas dependió también de la conformación de estos barrios y de su creciente población. Esta relación les permitía captar un gran número de usuarios, teniendo en cuenta que la mayor demanda del servicio de buses se encontraba precisamente en los nuevos barrios populares conformados por obreros y desempleados que buscaban conectarse diariamente con la ciudad formal.

En la década de 1970 se inició la ocupación informal de una amplia zona ubicada en el oriente de la ciudad, que corresponde hoy a las comunas 13, 14 y 15, sector conocido como el Distrito de Aguablanca. Ello se dio a través de una acelerada venta de lotes sin servicios a través de urbanizadores clandestinos y terratenientes interesados en obtener ganancias, además de varias tomas populares de lotes, aunque los terrenos no eran aptos para urbanizar. Asimismo, en el mismo sector y a través del programa de lotes con servicios, el Instituto de Vivienda de Cali (Invicali') localizó algunos proyectos de vivienda, aunque del programa sólo fueron entregados los lotes, pues el sector carecía de servicios públicos (Mosquera, 1984, p. 13).

En 1969 se creó la Oficina de Planeación Municipal, que pretendía crear planes de desarrollo que no se limitaran al diseño físico de la ciudad, incluyendo aspectos de desarrollo social, económico y urbano. El nuevo modelo se sintonizaba con las políticas de desarrollo planteadas desde el Gobierno Nacional que fortalecieron el papel de la planeación en los aspectos sociales y económicos, a partir de la reforma constitucional de 1968 (Vásquez, 2001, p. 284). Las nuevas ideas de planeación se vieron impulsadas, además, por la designación de Cali como sede de los vi Juegos Panamericanos de 1971, lo que implicaba adecuar la infraestructura de la ciudad para el evento deportivo.

Así se inició la formulación del primer plan de desarrollo de la ciudad, denominado Plan General de Desarrollo de Cali y su Área Metropolitana 1970-1985-2000. Las expectativas puestas en el Plan y la situación de segregación de la ciudad se evidencian en el prólogo realizado por el alcalde del momento, Carlos Holguín Sardi:

El crecimiento extraordinario, explosivo y analítico a que se vio la ciudad sometida durante los últimos lustros corrió aparejado de una especie de indolencia o derrotismo de nuestras gentes para afrontar el desafío que ese mismo crecimiento representaba. De ahí surgió no una ciudad, sino, como lo hemos dicho muchas veces durante estos meses, dos ciudades casi irreconciliablemente desunidas, desconocidas la una para la otra, manifestación trepidante

1 Institución creada en 1966 por el Concejo municipal, cuyo propósito era crear proyectos integrales de vivienda que permitieran erradicar tugurios, rehabilitar zonas deprimidas y legalizar asentamientos informales.

tiempo\&economía

Vol. 5 N. 2 - Julio - Diciembre del 2018 
de un déficit acumulado de soluciones y servicios y de un superávit creciente de esperanzas. Hay entre las dos ciudades un rasgo común: su ambición de progreso, su decisión de triunfo. Él ha sido espoliado con el pretexto de los compromisos internacionales que la ciudad debe cumplir en el año de 1971. En esta coyuntura histórica la aparición del Plan General de Desarrollo es afortunada y necesaria. (1971, p. s.p.)

Una de las problemáticas que según el Plan General de Desarrollo -PGD- afrontaba la ciudad, debido a su forma de crecimiento urbano, era el de los flujos encontrados de desarrollo urbano, uno de características centrípetas o centralismo físico-funcional y otro centrífugo o de expansión urbana. El flujo centrípeto era causado por la ubicación de la mayoría de servicios y lugares de trabajo en el centro de la ciudad, pues a pesar de su crecimiento, Cali seguía funcionando con un modelo antiguo, en el cual todas las vías municipales convergían en el centro. El flujo centrífugo era el generado por el crecimiento incontrolado de la ciudad desde la década de, que había llevado a la obsolescencia de sus servicios comunales y de su infraestructura. Estas dos situaciones significaban el aumento en los problemas de movilidad que sufrían los ciudadanos (PGD, 1971, pp. 15-16).

Sobre el transporte en modalidad de buses, el PGD indicaba una alta dependencia de la ciudad hacia este servicio debido al bajo nivel de ingresos de la mayoría de ciudadanos. Los datos recopilados en el PGD evidenciaron una estrecha relación no pavimento-bajos ingresos, pues los sectores que representaban los menores ingresos económicos, eran precisamente los que carecían casi por completo de vías pavimentadas.

El Plan concluía que la operación de los buses veía encarecidos sus costos debido al mal estado de las vías que incrementaba los tiempos de viaje y deterioraba los vehículos. Señalaba también que las rutas carecían de lógica, con recorridos muy extensos sin razón de serlo, desgastando innecesariamente los vehículos. También sobrecargaban algunas vías, como el caso de la Calle 15 (centro de la ciudad) por la cual transitaban 25 de las 27 rutas existentes en 1969. Estas afirmaciones del PGD desconocían el sentido que las rutas tenían para los transportadores; lo que para los expertos en transporte representaba un sin sentido, para los transportadores (conductores y dueños de empresas) representaba la oportunidad de transportar el mayor número de pasajeros, obteniendo la mayor ganancia posible en cada recorrido, sin importar las preocupaciones que el PGD planteaba. La falta de lógica institucional era, en realidad, la lógica económica de las empresas de transporte.

Algunas de las recomendaciones del PGD para mejorar el funcionamiento de los buses fueron: tratar de establecer rutas con recorridos directos, impedir que los buses entraran al centro de la ciudad, establecer paraderos para que los buses no se detuvieran en cualquier lugar; aunque no precisaba cuáles eran las estrategias para la organización del transporte, por lo cual terminó siendo más analítico que propositivo. Para Bonilla (2012), a partir del PGD se hizo más evidente "el divorcio entre el andar urbanístico y la generalidad de los planes (de desarrollo)" en Cali.

El Plan también asumió que para 1985, el nivel de viajes diarios por persona dentro de la ciudad se mantendría en un promedio de 1,6. Aunque la población aumentaría y las distancias también, los viajes al centro de la ciudad se reducirían, debido a la ubicación de áreas comerciales e industriales en zonas próximas a las áreas residenciales. Además, afirmaba que no habría un gran incremento de vehículos particulares per cápita; por el contrario, aumentarían 
las vías pavimentadas y las mejoras en la red vial, lo que optimizaría el sistema de transporte público haciendo más eficientes y convenientes sus rutas.

Aunque el PGD esbozó la construcción de un sistema de Tren Metropolitano que conectaría los municipios de Jamundí, Yumbo y Palmira con Cali, advertía que su construcción aún no era necesaria, "La ciudad de Cali no precisará de una solución masiva en el transporte colectivo hasta después del periodo 1980-1985 cuando la población sobrepase los 2.000.000 de habitantes" (1971, p. 224).

El único proyecto del PGD llevado a cabo fue el Plan Vial, plan de infraestructura favorecido por el Concejo y el Gobierno nacional, debido a la pronta realización de los Juegos Panamericanos. Las obras realizadas, aunque provocaron transformaciones urbanas importantes, sólo se limitaron al aspecto físico de la ciudad; por lo cual la pretensión de convertir el PGD en instrumento de políticas públicas no tuvo éxito. La promesa del alcalde Carlos Holguín, de disminuir la brecha entre las zonas periféricas surgidas informalmente y la ciudad formal, no se cumplió; por el contrario, la división establecida con la nueva Autopista Sur-oriental (ver mapa 1), materializó la división socio-económica entre el oriente y el resto de la ciudad y terminó por consolidar un modelo de distribución del espacio urbano denominado las dos ciudades: "la de los integrados y la de los excluidos en términos socio-espaciales" (Vásquez, 2001, p. 275). Es en esta división socio-espacial donde tomó mayor relevancia el transporte público, como mecanismo mediante el cual las dos ciudades se conectaban e interrelacionaban.

\section{Diagnósticos sobre el transporte: muchos debates y pocas soluciones}

A partir de la década de 1970, el transporte público colectivo en el país estuvo marcado por la creación, por parte del Gobierno nacional, del subsidio al transporte urbano a través del Decreto 1277 de 1971, medida que definió en gran parte la actuación de las empresas de transporte y su relación con el Estado. El subsidio se pagaba por cada vehículo en funcionamiento y tenía como finalidad, en un principio, cubrir el sobrecosto de la gasolina para que este valor no fuera trasladado a los usuarios en aumentos del pasaje.

La justificación para crear el subsidio era que, al hacer la relación entre los costos de operación de las empresas y el valor en el que se mantenía el pasaje, el negocio generaba pérdidas. Por lo cual, para no trasladar todo el aumento al usuario, el gobierno compensaba con el subsidio una parte del valor del pasaje. Éste se pagaba directamente a las empresas por cada vehículo afiliado de acuerdo con sus días de servicio en el mes: de 1 a 10 días no había derecho a subsidio; de 11 a 20 días se pagaba el 50\% del subsidio y de 21 a 30 días se pagaba el 100\% del subsidio (Presidencia de Colombia, Decreto 272 de 1972). Por día de servicio se entendía la realización de mínimo tres recorridos completos de una ruta asignada. Para el cobro, el gobierno suministraba un formulario que debía ser llenado por la empresa de transporte de acuerdo con las planillas de control y despacho de los vehículos. Modalidad que generó polémica ya que se hablaba de planillas alteradas, al marcar recorridos que los buses no realizaban. Otra modalidad era la circulación del bus sin importar la poca afluencia de pasajeros; aunque no se percibieran ganancias en el recorrido, el dueño del vehículo y la empresa salvaban el cobro del subsidio.

tiempo\&economía

Vol. 5 N. 2 - Julio - Diciembre del 2018 
162 La ciudad de los buses de colores: Empresas de transporte público, planes de desarrollo y crecimiento urbano en Cali (Colombia) 1969-1993

Mapa 1. Cambios en la ubicación de empresas de transporte (controles) entre 1964 y 1980

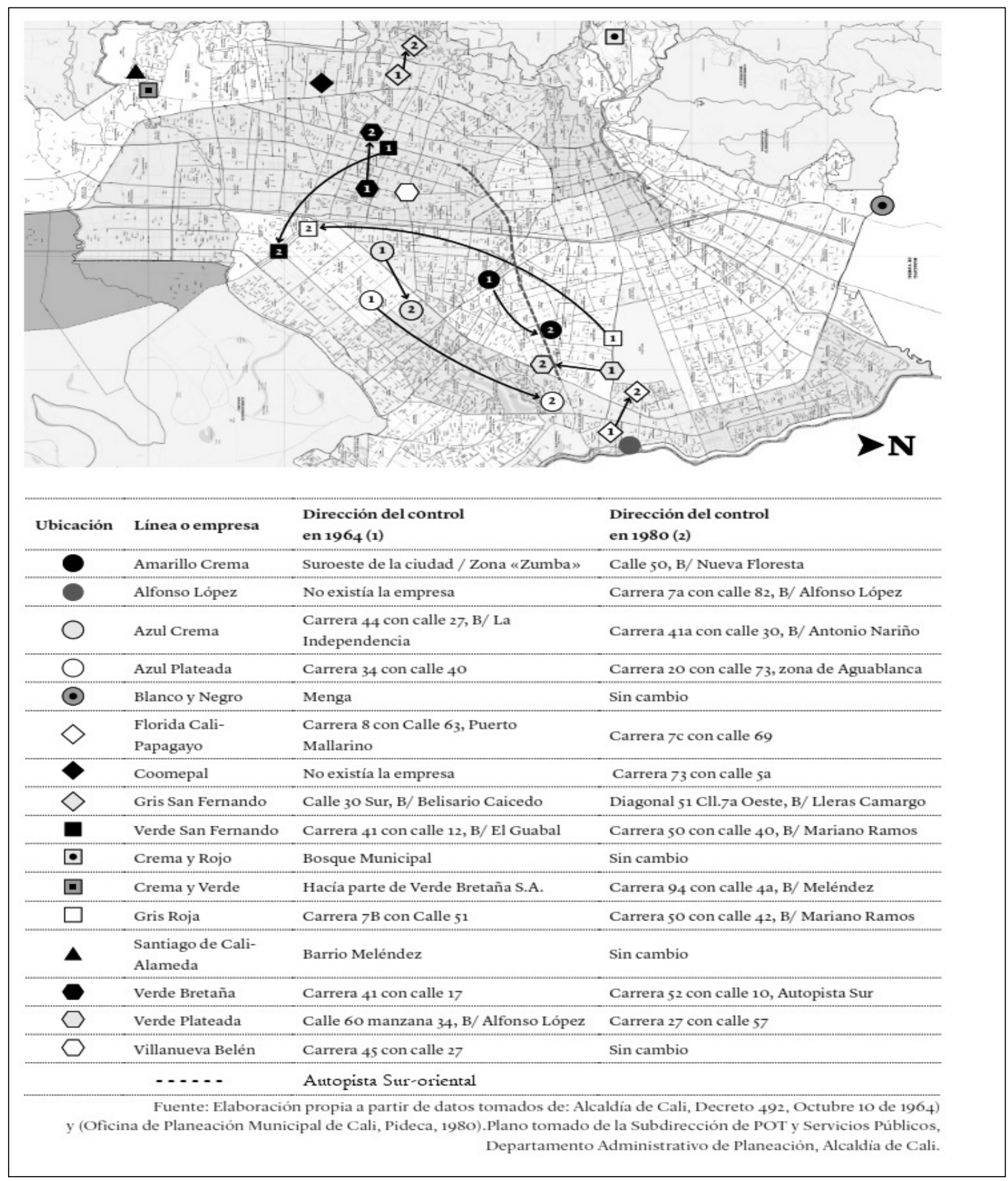


Además del subsidio para evitar grandes aumentos en los pasajes, el gobierno nacional buscó regular la operación de las empresas de transporte público mediante la creación de Instituto Nacional de Transporte (Intra) en 1968, como entidad ejecutora de las políticas nacionales en materia de transporte. Dos años después, mediante el Decreto Nacional 1393 de 1970, se autorizó al Intra para organizar y asesorar las empresas de transporte, asignar, modificar y cancelar rutas y fijar las tarifas del servicio. A partir de allí se crearon sedes regionales del Intra, incluida la regional Valle del Cauca ubicada en Cali.

Una de las primeras recomendaciones hechas por el Intra para Cali, fue la creación de una Secretaría de Tránsito, llevada a cabo por el Concejo mediante Acuerdo 19 de 1972. Las razones expuestas para su creación eran el dualismo de funciones que se presentaba entre las Direcciones Departamental y Municipal de Tránsito; funciones que ahora, se esperaba, fueran concentradas en la nueva entidad.

Otra de las tareas realizadas por el Intra, fue la publicación en 1973 del Estudio de Reestructuración de Rutas Urbanas para Cali, en el que se realizó un inventario del sistema de transporte público, estableciendo cuáles eran los recorridos que realizaban los buses en cada una de las 32 rutas existentes en 1972, las condiciones de las vías, tiempos de recorrido y formas de operación de las empresas; con el fin de hacer las recomendaciones necesarias para mejorar el sistema de transporte público en la ciudad.

El análisis evidenció una diferencia entre las rutas autorizadas y las recorridas, principalmente en los barrios populares. Esta práctica obedecía, según el informe, no a la búsqueda de calles pavimentadas sino a la búsqueda de pasajeros cerca a sus domicilios, lo que hacía que los buses realizaran muchas vueltas por un mismo sitio. Asimismo, la tendencia de los buses era pasar lo más cerca posible al centro, para dejar al usuario muy cerca de su destino; prácticas que se debían modificar para agilizar el transporte (1973, p. 55). Además, el tiempo que perdían algunas rutas en los barrios populares, buscaba ser compensado con su recorrido por vías principales; recorridos que, en gran parte, no estaban autorizados, pero obedecían a un sentido lógico de agilización del transporte (Intra, 1973, p. 10).

Estas tendencias en los recorridos permiten evidenciar una alta operación de los buses por fuera de las rutas aprobadas, con lo cual se puede afirmar que, aunque las empresas y rutas estaban legalmente constituidas, en la práctica actuaban en un alto porcentaje por fuera de lo que las autoridades de tránsito les permitían; buscando incrementar sus ganancias. Estas formas de operar favorecían la práctica de los usuarios de elegir las rutas que los recogieran y dejaran más cerca de sus lugares de origen y destino, con lo cual las empresas de transporte, que operaban bajo una lógica de mercado, desestimulaban la aceptación de los ciudadanos hacia medidas como la peatonalización del centro o la instalación de paraderos exclusivos que evitaran a los buses detenerse constantemente en busca de pasajeros.

Otra de las observaciones hechas por el informe era la necesidad de adecuar las vías para evitar los recorridos "tortuosos" y agilizar los tiempos, principalmente en el sector oriental, donde se ubicaba gran parte de los barrios populares surgidos de manera informal. A pesar de la dificultad en las vías, se señalaba que la ciudad estaba servida adecuadamente en todas sus zonas por el servicio de buses. Las rutas que tenían el menor porcentaje de recorrido por vías pavimentadas, eran las que ubicaron sus controles en la zona oriental, lo que muestra que el pavimento no era un factor de decisión a la hora de establecer el recorrido de una ruta. Por el 
contrario, si tenemos en cuenta la relación no pavimento-bajos ingresos establecida en el PGD y la dependencia de los sectores populares hacia este servicio, la precariedad de algunas zonas podía significar el éxito de algunas rutas al tener mayor oportunidad de captar usuarios. Prueba de ello es que, como se aprecia en el mapa 1, la mayoría de empresas ubicaron sus terminales (controles) en las zonas periféricas de la ciudad. Para 1980 varias empresas habían desplazado sus instalaciones mucho más al oriente y hacia las laderas de la ciudad, en comparación con la ubicación que tenían en 1964.

En cuanto a la estructura administrativa de las empresas de transporte, se calificaba como inadecuada y poco funcional. Según el Intra, a pesar de la desorganización financiera de las empresas, estas subsistían debido a la rentabilidad del negocio del transporte en la ciudad. Para 1972, los ciudadanos realizaban diariamente 1.200.000 viajes dentro de la ciudad, de los cuales 900.000 eran realizados en bus. La mayoría desde la zona oriental hacia el centro. A pesar de las recomendaciones y el amplio diagnóstico realizado por el Intra, la propuesta de reestructuración de rutas, que era el máximo objetivo del estudio, se desconoce, pues de los cinco informes que componían el estudio, solo se conservan cuatro en la actualidad.

En un documento de 1978 realizado por la Secretaría de Tránsito denominado Evaluación transporte público urbano en Cali se evidencia que entre la realización del estudio del Intra y este nuevo informe, la situación del transporte público no tuvo cambios significativos. El nuevo estudio era muchos más crítico respecto a la operación de las empresas y el papel que cumplía el Intra. El dualismo de funciones que había expuesto el Intra en su momento para crear la Secretaría de Tránsito, era ahora manifestado por la misma Secretaría (1978, p. 32). Señala el texto que a pesar de las funciones que el Estatuto Nacional de Transporte había asignado al Intra, esta entidad incumplía sus funciones de organización y vigilancia; lo que obligaba a la Secretaría a cumplir funciones que no le correspondían, como era el tema de los estudios técnicos para asignación de rutas a las empresas (1978, p. 23).

Las principales conclusiones a las que llegó la Secretaría fueron que el sistema de transporte urbano se caracterizaba por "un ánimo exclusivo de lucro" sin tener en cuenta la calidad del servicio. Las rutas habían nacido sin planificación ya que las empresas, de acuerdo con el crecimiento de la ciudad, iban solicitando nuevas rutas o prolongación de las existentes. Es decir que, aunque en teoría la decisión sobre los recorridos de los buses le correspondía al Intra, en la práctica eran los directivos de las empresas de transporte quienes decidían hacia donde enviar las rutas y que, según el mismo informe, eran personas con conocimientos empíricos a las que interesaba unicamente las ganancias; decisiones que posteriormente eran aprobadas por el Intra.

Sin embargo, estos informes omitieron el tema de las formas de contratación de los conductores, como uno de los factores de deficiencia en el transporte. Los conductores obtenían sus ganancias de acuerdo con el número de pasajeros que lograban captar diariamente, lo que originó la competencia de buses por las calles de la ciudad, buscando añadir un pasajero más a la ganancia del día; problemática conocida como "la guerra del centavo" y causante de la mayoría de accidentes de buses en las vías. 


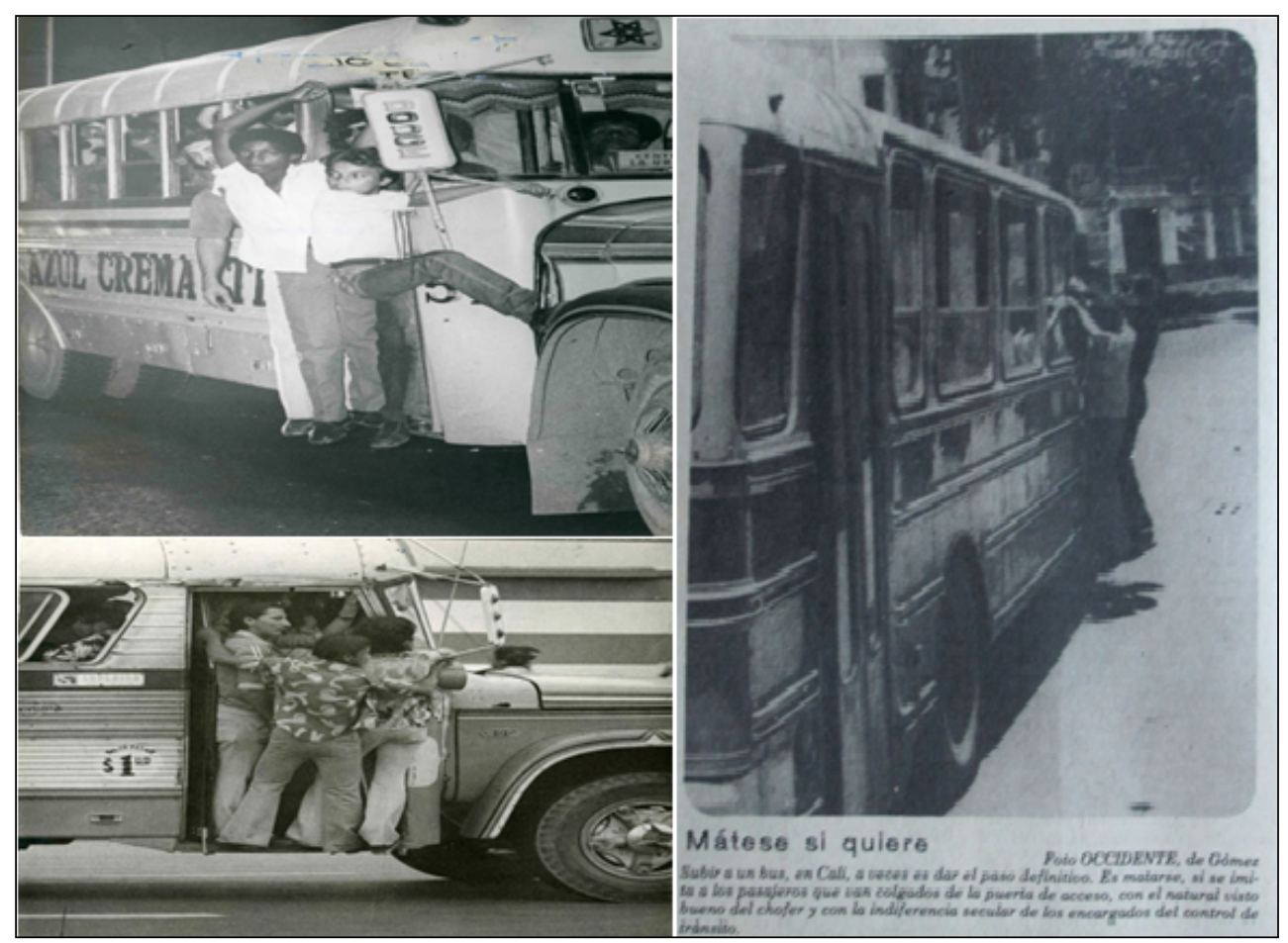

Fuente: Diario Occidente (1977), Archivo del patrimonio fotográfico y fílmico del Valle del Cauca $(1979,1983)$

Los buses que operaban en una empresa podían ser de propiedad de la empresa, de los socios o de los afiliados; estos últimos eran personas que poseían uno o varios vehículos y que mediante un porcentaje de pago tenían derecho a que sus vehículos funcionaran en la empresa; los afiliados eran en algunos casos conductores de sus propios vehículos lo que les permitía obtener un poco más de ganancia al reducir costos de operación. Las empresas por su parte, tenían pocos vehículos de su propiedad.

Jaramillo (1985) señala que la mayoría de los conductores no eran propietarios sino asalariados; las funciones de las empresas eran mínimas y cumplían muy poco con su papel de regular el servicio; por el contrario, el beneficio que recibían al tener el control de las rutas llevó a una relación de dependencia de los propietarios de buses hacia las empresas, que permitió el cobro de rentas y la creación de mercados cautivos para los insumos de los vehículos. A esto se añadió el gran atraso de las condiciones laborales de los conductores, ya que los vínculos se establecían no con la empresa, sino entre el trabajador y el propietario del vehículo:

Sus jornadas son enormemente prolongadas, tienen muy poca estabilidad, el trabajo es muy intenso y desgastador, pues deben simultáneamente cobrar y conducir en un tráfico endemoniado, compitiendo con sus colegas por los pasajeros de los cuales depende su salario. Carecen en la práctica de prestaciones sociales como la jubilación, horas extras, no reciben ingresos cuando el vehículo se avería, y por el contrario deben realizar gratuitamente tareas de reparación y de mantenimiento. Y sin embargo... el salario global que estos conductores pueden reunir es comparativamente alto, entre dos y dos y media veces el salario mínimo. (Jaramillo, 1985, p. 288) 
Por su parte, las leyes vigentes sobre contratación laboral en el país señalaban:

El contrato de trabajo verbal o escrito, de los choferes asalariados del servicio público, se entenderá celebrados con las empresas respectivas, pero para efecto de pago de salarios, prestaciones e indemnizaciones, las empresas y los propietarios de los vehículos, sean socios o afiliados, serán solidariamente responsables. (Congreso de Colombia, Ley 15, 1959)

En la práctica, ni la empresa ni el dueño del vehículo asumían las responsabilidades de ley. Las ganancias superiores al promedio, hacían que los conductores prescindieran de sus derechos laborales y que se normalizaran las prácticas de contratación que favorecían las ganancias de las empresas en detrimento de la calidad de vida de los conductores. Todo ante la indiferencia de las autoridades nacionales, quienes optaron por no enfrentarse a los empresarios del transporte que adquirían cada vez mayor poder en el país. Poder que les permitía incluso, manejar abiertamente un esquema de contratación contrario al ordenado por las leyes vigentes del país, debido a que su fuerza residía en manejar un servicio imprescindible para los ciudadanos. Una nota publicada en la Revista Semana en 1982 da cuenta de ello.

Cuando los dueños de los buses se ponen inquietos, las ciudades corren el riesgo de quedarse quietas. Esto es lo que hace de Conaltur -Confederación Nacional de Transporte Urbano- un gremio poderoso que gana siempre que se sienta a la mesa de negociaciones, porque antes de hablar, deja entrever su arma contundente: la posibilidad de paralizar el transporte. (Revista Semana, agosto 30 de 1982)

A inicios de los años ochenta el Gobierno nacional, a través del Intra, inició el desmonte progresivo de los subsidios al transporte, justificado en las irregularidades que se presentaban en su cobro y en la mala calidad del servicio. Las críticas hacia los subsidios aumentaron, debido a las continuas alzas que los transportadores exigían al Gobierno Nacional y a denuncias sobre posibles fraudes que se estaban cometiendo en su cobro.

La base del problema está en que en Colombia, como es común en toda América Latina, el transporte colectivo es un negocio privado, cuyos dueños se ponen de acuerdo con el gobierno en una premisa básica; se trata de una empresa que da pérdidas, y que debe por tanto ser subsidiada... El sistema de subsidio da lugar a irregularidades grandes. Por ejemplo, un lego en la materia se puede preguntar si es factible el caso de un dueño de bus que lo deje guardado en un garaje, le cobre al gobierno el subsidio y se ponga así una renta mensual de $\$ 40.000$ o \$60.000 sin mover un dedo. La duda se acentúa si se tiene en cuenta la total insuficiencia del sistema de control que tiene el gobierno para los recorridos, que consiste en que cada bus tiene una cartulina, que es sellada al principio y al final del recorrido en las casetas de control de las terminales. ¿Cuánto vale un sello? ¿Dos cervezas? ¿Doscientos pesos? Hay una experiencia que da una idea. Cuando Miguel Urrutia fue director de Fedesarrollo y quiso estudiar el problema de los buses, optó por una medida empírica para penetrar en ese laberinto. Hizo que la entidad, de manera camuflada, comprara un bus, le contratara un chofer y lo pusiera a prestar servicio como cualquier otro. Día a día le seguía la trayectoria al bus, hasta que éste se descompuso y se varó. Cuando el chofer vino a comunicarle la noticia añadió: "Pero no se preocupe, doctor, que la cartulina sí está viajando". (Revista Semana, agosto 30 de 1982) 
Aunque el gobierno solicitaba que las empresas de transporte mejoraran el servicio, adquirieran mejores vehículos, disminuyeran el sobrecupo y colocaran en funcionamiento todo el parque automotor del que disponían; los transportadores insistían en que el subsidio sólo compensaba tres recorridos y el resto se trabajaba a pérdida, por lo cual para obtener ganancias los buses debían ir llenos y adquirir vehículos nuevos no era un buen negocio, ya que con los viejos se obtenía la misma ganancia. Por ello, para 1982 el Intra creó una nueva modalidad de transporte en el país: el Transporte Sin Subsidio (TSS). Con ello, las empresas cobrarían la tarifa plena que les garantizaba ganancia en todos los recorridos y el Gobierno disminuía el gasto en subsidios.

Los buses que prestarían el nuevo servicio, según la Resolución 342 de 1982, debían cumplir con las siguientes características: sólo se aceptaban vehículos modelo 1981 y posteriores, debían tener silletería de lujo, puertas más amplias, contar con un aviso frontal luminoso con las letras Tss y ser pintados de color amarillo y verde para diferenciarse claramente de los buses subsidiados, que continuarían portando los colores tradicionales de cada empresa.

Una de las características más sobresalientes de los buses de Cali eran sus colores, pues cada empresa pintó sus buses con una gama de colores que la diferenciara de las otras y, además, en la gran mayoría de los casos, el nombre de las empresas se correspondía con los colores que asignaba a sus vehículos: Amarillo Crema, Crema y Rojo, Blanco y Negro, Azul plateada, Gris roja, Papagayo, Verde plateada, etc.

Los Tss sólo podían llevar 20 personas de pie. Las empresas podían cambiar su parque automotor y remplazarlo completamente por buses que cumplieran con las nuevas características o podían seguir funcionando en las dos modalidades: Tss y subsidiado (Intra, Resolución 342 de 1982). Sin embargo, para este momento ya se dudaba de los efectos positivos de la medi$\mathrm{da}$, teniendo en cuenta que el desmonte de los subsidios significa principalmente el aumento en las tarifas a los usuarios:

A pesar de las distorsiones y de los malos usos a que da lugar por parte de algunos transportistas, el subsidio es la única medida viable para que los colombianos puedan ir de un lado a otro sin que les cueste más de lo que pueden pagar... EI TSS es el ejemplo de lo que pasaría si prosperara el intento de desmontar este sistema: son $\$ 7.50$ más que salen del bolsillo del pasajero, mientras que lo único que cambia del bus es el color. (Revista Semana, agosto 30 de 1982)

Sin embargo, a su llegada a la presidencia del país en 1983, Belisario Betancur continuó el desmonte progresivo de los subsidios. Mediante Decreto 3615 de diciembre del mismo año, estableció una rebaja en los subsidios entregados a cada vehículo y lo condicionó al modelo de fabricación de los buses: a mayor antigüedad menor subsidio. Los buses más nuevos, con modelos entre 1974 y 1978 que funcionaran con sistema diésel recibirían mayor subsidio que los de sistema a gasolina. Los recorridos diarios que debía realizar cada bus para obtener el subsidio también se aumentaron; para Cali fueron aumentados de tres a cinco. También se anunciaba que, a partir el 1 de enero de 1984, los buses de modelos posteriores a 1978 serían retirados del programa de subsidios e ingresarían al de tss (Presidencia de Colombia, Decreto 3615 de 1983). En noviembre de 1984 el presidente Betancur emitió el Decreto 2775 que retiraba del sistema de subsidios a los buses de modelos entre 1974 y 1977 y los incluía en el sistema TSS. Las condiciones iniciales para el cobro de tarifa plena por parte de los Tss habían 
desaparecido y las mejoras en el servicio nunca llegaron. Los buses con subsidio eran escasos y las tarifas subían. Los buses verde-amarillos continuaban aumentando.

En 1986, fue aprobada en el país la elección popular de alcaldes. A partir de allí también se inició una serie de cambios en cuanto a las funciones de los municipios tendientes a la descentralización del Estado y a asignar mayor autonomía a los municipios y regiones. Una de estas medidas fue eliminar el Intra y trasladar sus funciones a las administraciones municipales (Decreto 80 de 1987), con la cual también desaparecía la entidad reguladora de los subsidios en el país.

Sin embargo, la desaparición definitiva de los subsidios no fue obra de presidentes ni de alcaldes; fue la Corte Constitucional quién puso fin al subsidio. Primero declarando inexequible el Decreto 1277 de 1971 y el Decreto 588 de 1978, impidiendo pagar subsidios diarios por vehículo a las empresas. Los subsidios se intentaron revivir con la Ley $2^{\text {a }}$ de 1989 , pero esta fue demandada señalando que el Congreso estaba decretando "gratificaciones" del poder público a particulares y que el subsidio no estaba sujeto a planes, ni era un derecho de los transportadores. La demanda fue aceptada y la Ley declarada inexequible, con lo cual el subsidio llegó a su fin (Corte Suprema de Justicia, Sentencia 96 de 1989).

A la par con las discusiones sobre los problemas del transporte público tradicional, aparecen en la ciudad las primeras propuestas para renovarlo, como solución a los problemas que se venían acumulando desde décadas atrás. Aunque los análisis planteaban la necesidad de municipalizar el transporte, acabando con la gran cantidad de empresas que operaban con ánimo de lucro un servicio que debía ser garantizado por el Estado; las propuestas reales presentaron grandes problemas de inversión que las hicieron caer en el olvido, mientras las empresas de buses continuaban creciendo.

La primera de ellas surgió con la realización del Plan Integral de Desarrollo de Cali y su área de influencia (Pideca). El diagnóstico sobre la ciudad realizado para el plan, evidenció que entre el PGD y el Pideca el panorama de la ciudad no había sufrido mayor variación. Además de continuar su crecimiento incontrolado, se hacía aún más evidente el panorama de dos ciudades divididas por una autopista.

Para su análisis sobre el transporte, el Pideca, además de recopilar información en los barrios, tomó como base los trabajos realizados en la década de 1970 por el Intra y la Secretaría de Tránsito. Por ello, las cifras y datos arrojados por el Pideca, coinciden ampliamente con los diagnósticos anteriores; principalmente el realizado en 1978. En cuanto a las conclusiones, el Plan reiteraba la crítica al modelo de transporte con ánimo de lucro y la inconveniencia de las rutas establecidas.

Afirmaba que el trasporte se había convertido en una pirámide en cuyos niveles inferiores se encontraban los usuarios, quienes sostenían con las tarifas a los conductores y los vehículos; los conductores por su parte sostenían, con su mala remuneración y sus largas jornadas, a los propietarios de los vehículos. Finalmente, los empresarios en la cúspide, eran sostenidos por todos los anteriores, pagando únicamente los gastos de administración de la empresa, pues del total de buses que funcionaban en la ciudad, sólo el 2,15\% pertenecían a las empresas de transporte; el resto era propiedad de los socios o afiliados (Pideca, Documento 17, 1980, p. 82). 
Las empresas transportadoras se encontraban atrasadas en sus métodos de administración y operación. Esto era atribuido a que los empresarios, por lo regular, tenían conocimientos empíricos sobre el manejo empresarial; ya que generalmente habían escalado posiciones en el sector luego de ser conductores o afiliados. Para el Pideca, el interés prioritario de estos dirigentes era obtener el mayor número de rutas, buscando aumentar las ganancias. Sin importar las precarias condiciones laborales de los conductores, que redundaban en un mal servicio a los ciudadanos:

- No tienen salario fijo.

- Tienen pocas prestaciones sociales.

- Su nivel cultural es bajo.

- Además de conducir, ejecutan otras labores como cobrador y mecánico.

- No tienen jornada fija de trabajo y por lo general esta, es inhumana.

- No existe la carrera de conductor, cualquier persona consigue un pase y puede manejar un vehículo público.

- No tienen bien definido el patrón. [Jefe]

- Existe la tendencia de asignar un conductor a un vehículo.

- Tienen un sistema de pago a base de centavos por pasajero transportado, altamente inconveniente.

- Son los que tienen una jornada larga más constante.

- Se les acusa de ser los únicos causales de los problemas de tráfico (Pideca, tomo 43, 1980, p. 6.18).

Las condiciones en las cuales operaban las empresas eran favorecidas por el número creciente de usuarios, debido a la tasa de crecimiento de la ciudad. Según el Plan, las continuas obras de infraestructura y servicios causaban un efecto multiplicador de la población que en su mayoría llegaba a ocupar los sectores populares e informales de la ciudad; estas zonas continuaban significando para las empresas, las mayores oportunidades de obtener rutas. La ubicación que había adquirido la mayoría de terminales, en la zona oriental, significó para sus habitantes una garantía de conexión y de inserción en las oportunidades económicas y laborales que ofrecía la ciudad formal (Aprile-Gniset, 1992, p. 627). El caso del barrio El Retiro es muestra de ello:

Al inicio se sufrió inhumanamente, las gentes más afectadas fueron las del barrio El Retiro, porque los terrenos entregados eran lagunas, cuando llovía era el primero en inundarse, donde les tocó prácticamente hacer el relleno de todo el barrio. Se alumbraban con vela, en el primer año no había alcantarillado, eran solamente letrinas y sanitarios que se colocaron en el canal de la calle 48... En materia de transporte, no eran sino dos las empresas que prestaban el servicio (Azul Crema y Alfonso López), en muchas ocasiones el transporte quedaba suspendido por el mal estado de las vías cuando llovía. (Centro de administración local integrada, Comuna 15, 2003)

tiempo\&economía

Vol. 5 N. ${ }^{\circ} 2$ - Julio - Diciembre del 2018 
Incluso en algunas ocasiones, además del transporte, las empresas suplieron otro tipo de necesidades:

Los terrenos carecían de servicios públicos, los primeros habitantes satisfacían sus necesidades de agua potable transportándola en vasijas al hombro desde el control de la Azul Plateada, algunos hicieron de esta necesidad un negocio vendiendo los 5 galones de agua a \$20 pesos, fue entonces cuando la comunidad se unió y trajo el agua con mangueras naciendo así las pilas comunitarias El transporte se hacía en camperos, los primeros buses fue de la empresa Azul Plateada (sic). La energía era suplida por velas. (Centro de administración local integrada, Comuna 14, 2003)

Las historias de cómo se conformaron los barrios informales evidencian la importancia de los buses en los procesos de configuración de estas zonas, contribuyendo incluso, a legitimarlas como parte de la ciudad formal.

Imagen 2. Buses transitando por el oriente de Cali. Años ochenta

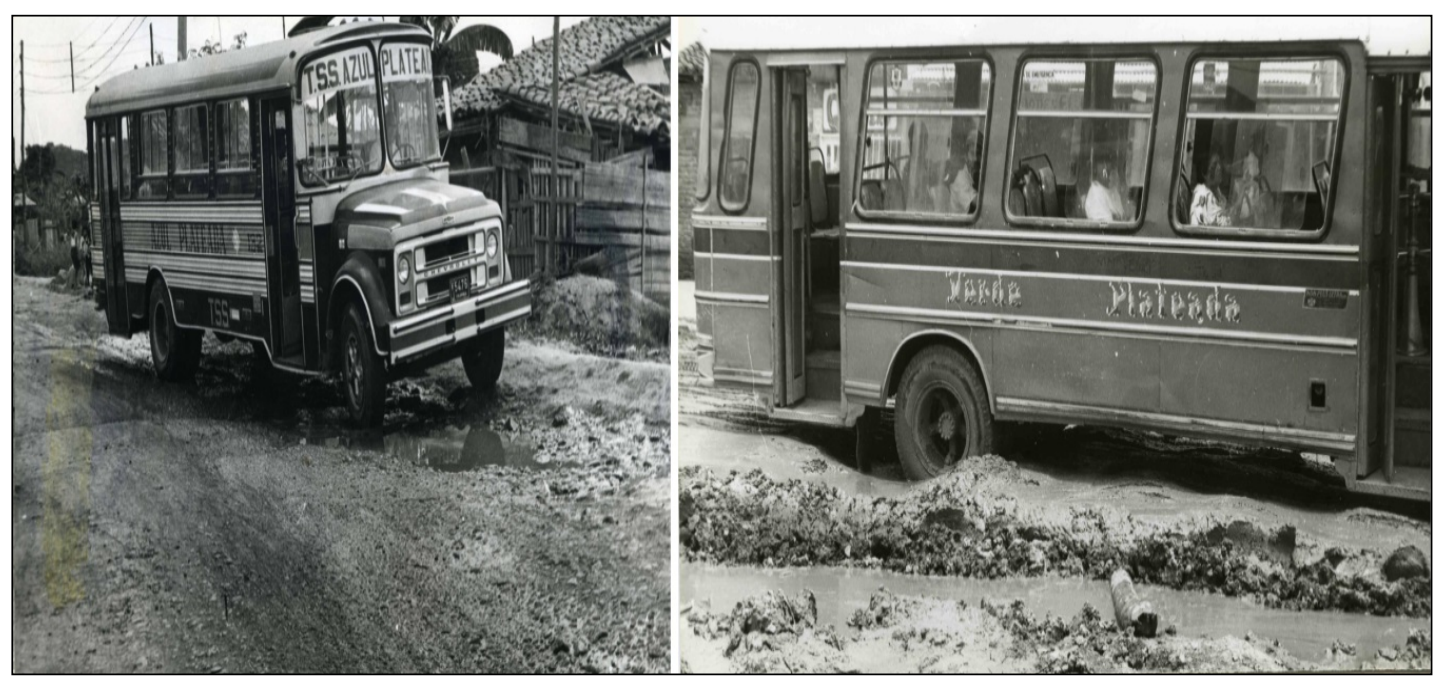

Fuente: Archivo del patrimonio fotográfico y fílmico del Valle del Cauca $(1986,1990)$

De otro lado, ante el panorama referido sobre el transporte colectivo de la ciudad, el Pideca optó por no proferir un plan de organización de este sector; señalando que cualquier intento sería insuficiente ante la actuación dual y descoordinada de las autoridades del transporte, cuyas decisiones se encontraban sujetas a lo que lograran negociar con los empresarios. Por ello la recomendación era buscar estrategias que permitieran al municipio ir adquiriendo progresivamente el control del sistema de transporte de la ciudad. Por último, el Pideca recomendaba iniciar estudios para la adopción de un sistema de transporte masivo, contribuyendo a los trabajos realizados por la Compañía Promotora de Transporte Masivo del Valle (Protrans) (Pideca, tomo 43, 1980, p. 6.45).

Protrans fue constituida el 5 de junio de 1979, impulsada por el Acuerdo 41 de 1978, mediante el cual el Concejo Municipal autorizó al Alcalde para crear las empresas que dieran solución a los problemas de transporte de la ciudad y su área de influencia. Un año después, para 
impulsar los proyectos sobre transporte que se llevarían a cabo, el Concejo de Cali mediante Acuerdo № 2 de 1980, destinó la vía del ferrocarril que atraviesa la ciudad de sur a norte, cómo área de reserva para el futuro sistema de transporte masivo que se llegara a implementar.

Por la misma época, la idea de construir un sistema masivo de transporte ya se estaba discutiendo en las ciudades de Medellín y Bogotá. En 1977 fue incluido en el Plan Metropolitano de Medellín un proyecto de tren metropolitano. En el mismo año, la ciudad de Bogotá inició los estudios para la construcción de un tren metropolitano y un año después constituyó la empresa Metro. La disputa por los recursos nacionales para el proyecto terminó con la llegada de un antioqueño a la presidencia del país, Belisario Betancur, que inclinó la balanza hacía Medellín (Acevedo, Salazar y Castañeda, 1993). Como señalan Ortiz y Villarreal (2016) el exacerbado regionalismo político que ha caracterizado al país, contribuyó a las pujas por los dineros públicos para infraestructura de transporte y terminó concentrando los recursos en obras locales.

A pesar de surgir en un marco local, Protrans se concibió para responder a las necesidades del transporte en el orden departamental. El documento Términos de referencia para una propuesta técnica sobre corredores de transporte rápido masivo para Cali y su área inmediata de influencia, realizado en 1981 por Protrans, señalaba que sus funciones eran planear, desarrollar y operar un sistema de transporte rápido masivo que uniera a Cali con los municipios vecinos y se proyectara hacia otras regiones, utilizando la ya existente vía del Ferrocarril del Pacífico. Mediante este documento, la entidad definió las bases para el concurso de méritos que adelantaría, con el fin de seleccionar una propuesta técnico-económica de corredores de transporte masivo para conectar, en un primer momento, a Cali y Palmira.

Dos años después, en 1983, Protrans publicó un nuevo documento: Estudio de posibles soluciones para el transporte público masivo en el área del valle geográfico del Río Cauca, realizado con la firma italiana Ansaldo Trasporti S.P.A en el marco del Acuerdo de cooperación técnica internacional entre Colombia e Italia, que permitió el aporte financiero del Ministerio de Relaciones Exteriores del país europeo al proyecto (Cancillería de Colombia, 1971).

Este nuevo estudio buscaba soluciones para el transporte de carga y pasajeros, dando especial importancia a la conexión entre Cali y su salida al mar Pacífico a través de Buenaventura. El transporte de carga fue priorizado y luego se pasaría a implementar el de pasajeros; pero lo que se buscaba era integrar las dos modalidades en un solo sistema de transporte. Protrans buscaba utilizar la red ferroviaria ya existente en el departamento y la ciudad para minimizar los costos de inversión.

Con la ayuda técnica y económica de la firma italiana, Protrans desarrolló cuatro propuestas de intervención de la red ferroviaria: intervención mínima, rehabilitación en unos puntos y nueva construcción en otros, rehabilitación integral de toda la vía o reconstrucción de toda la red (1983, pp. 2-3). El análisis final concluyó que ninguna de las alternativas arrojaba resultados económicos aceptables; por ello la decisión se veía orientada hacía una inversión de "costo mínimo", que justificara económicamente el funcionamiento del ferrocarril, de bajo costo energético, estratégica para el transporte pesado del país y susceptible de ser utilizada para el transporte de pasajeros.

Finalmente se decidió rehabilitar el tramo Cali-Buenaventura para transporte exclusivamente de carga, procurando las mínimas inversiones, y habilitar el tramo Cali-Palmira para el 
transporte de carga y pasajeros. Este último tramo contemplaba la duplicación de la vía férrea, pero se haría paulatinamente de acuerdo con la demanda que se fuera generando, postergando las obras más costosas para después del año 2000. El análisis costo-beneficio fue el que señaló la priorización del transporte de carga, por encima del de pasajeros, al considerar que este representaba rentabilidad económica, mientras que el de pasajeros tenía un carácter esencialmente social y territorial.

Aunque Protrans fue creado para buscar soluciones de transporte para la población y principalmente para la de menos recursos, no logró armonizar los intereses económicos con las necesidades de la población, por lo cual, para ser viable, el nuevo proyecto debía continuar funcionando, al igual que las empresas de transporte público, bajo la lógica de la rentabilidad económica.

Sin embargo, el proyecto de transporte masivo nunca se realizó, se desconoce que ocurrió con la Compañía, que labores desarrolló en años posteriores y por qué sus propuestas no tuvieron mayor repercusión en la ciudad y en el departamento. Así mismo se desconoce las razones que llevaron a su desaparición. Los dos informes mencionados constituyen los únicos documentos que se encontraron sobre Protrans.

Aunque todas estas cuestiones parezcan propias del ámbito local y regional; es ingenuo desconocer que el poco éxito de Protrans, estuvo también relacionado con las decisiones tomadas sobre el transporte para otras ciudades del país y con los proyectos que sí se llevaron a cabo. La precariedad de los proyectos de infraestructura en el país y la conformación de varios centros urbanos de importancia, llevó a una competencia por los recursos, en la cual Cali no salió muy bien librada.

\section{Que vuelva el color}

A partir de la promulgación de la Constitución de 1991, la planeación pasó a ser considerada como un proceso participativo en el cual debían estar involucrados todos los actores sociales; por lo cual los planes ya no serían realizados exclusivamente por las instituciones locales o regionales de planeación, sino que debían pasar por un proceso de socialización y aprobación en diferentes instancias para ser llevados a cabo, con lo cual los planes de desarrollo como habían sido concebidos entre las décadas del setenta y ochenta desaparecieron, y a partir de 1997 se convirtieron en Planes de Ordenamiento Territorial.

Durante la década de 1980, con la entrada paulatina de buses a la modalidad Transporte Sin Subsidio -TSS-, las empresas caleñas fueron unificando el color de los buses que se iban incluyendo en esta modalidad. Como ya se ha mencionado, los buses tss debían ser pintados de verde y amarillo. Aunque según lo ordenado por el Intra, estos debían tener otras especificaciones físicas y un modo de operación distinta; en la práctica por lo único que terminaron diferenciándose de los buses subsidiados fue por el color, debido a la inclusión al sistema tss de modelos antiguos.

A inicios de los noventa, cuando desapareció el subsidio, la mayoría de buses de la ciudad habían adquirido los colores del Tss. Por ello, mediante el Decreto 08 de 1993, el alcalde Rodrigo Guerrero ordenó a las empresas adquirir nuevamente para sus vehículos los colores que habían sido tradicionales hasta la década del setenta: "Con el objeto de que los usuarios dis- 
pongan de una facilidad visual para distinguir y escoger el servicio deseado sin someterse a las traumáticas confusiones generadas por la uniformidad en los colores distintivos existentes".

Para adoptar la medida, las empresas tenían un plazo de 18 meses; sin embargo, tres meses después y a solicitud de los empresarios, el plazo fue ampliado a cinco años contados a partir del 8 de enero del mismo año (Decreto 576 de 1993). Luego del vencimiento del plazo de cinco años, en 1998, los empresarios solicitaron al alcalde Ricardo Cobo un nuevo plazo de seis meses para cumplir la disposición. Plazo que, como era costumbre con las solicitudes de los empresarios, fue aceptado y aprobado mediante Decreto 614 de 1998.

El asunto del cambio en los colores de los buses, es sólo un ejemplo más del poder que llegaron a adquirir los empresarios en las decisiones sobre transporte que se tomaban en la ciudad. Las modificaciones y propuestas en el funcionamiento del transporte público e incluso en el tránsito general de la ciudad, en gran parte debían su éxito o fracaso a la receptividad que tuvieran de parte de este gremio. Para una síntesis de las acciones llevadas a cabo por el gobierno nacional y local por periodos y número de empresas ver el anexo.

\section{Imagen 3. Dos buses de la empresa Gris San Fernando: A la derecha con colores de TSS y a la izquierda} con colores tradicionales de la empresa

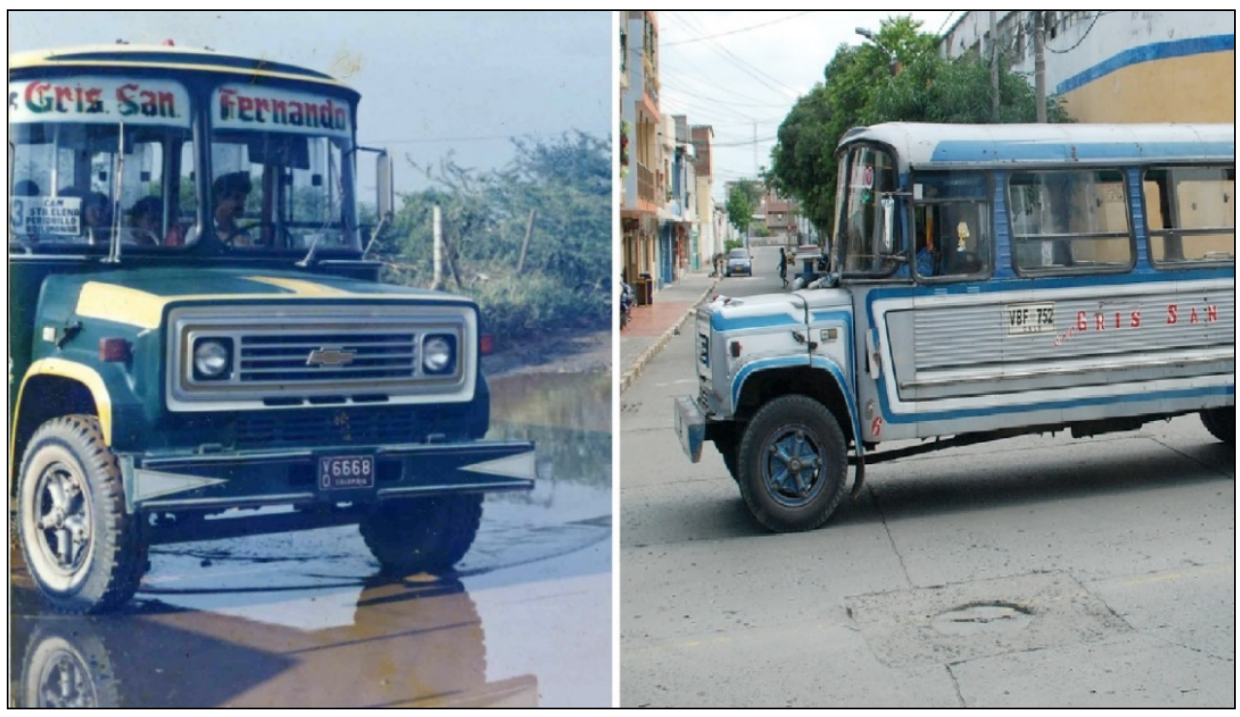

Fuente: Archivo del patrimonio fotográfico y fílmico del Valle del Cauca $(1993,2000)$

\section{Conclusiones}

Este documento ha pretendido esbozar algunas de las problemáticas surgidas de la relación entre el transporte público, las instituciones estatales y la ciudadanía caleña entre las décadas del setenta y el noventa del siglo xx, buscando, además, evidenciar la forma en que estos tres sectores se relacionaron y contribuyeron, de diversas maneras, al crecimiento y desarrollo de la ciudad.

Las políticas locales se analizaron a partir de los planes de desarrollo (PGD y Pideca) formulados para Cali, que a pesar de incluir elementos, no sólo de crecimiento físico sino también 
socio-económicos, no lograron mayor impacto en las formas de urbanización de la ciudad, la cual creció con un gran componente de informalidad y a través de formas precarias de construcción y financiadas por las familias que requerían una vivienda, en sectores no aptos para urbanizar. Los planes de desarrollo sólo impactaron la ciudad a través de algunas obras de infraestructura, dejando de lado la reducción de la desigualdad social que era expuesta como uno de sus grandes propósitos.

Las urbanización informal creció en relación estrecha con el desarrollo del transporte público, cuyas empresas -que operaban bajo una lógica de lucro económico- eran uno de los primeros servicios públicos que llegaba a los barrios marginados de reciente creación, convirtiéndose en la garantía de conexión de sus habitantes con la ciudad formal y las oportunidades laborales.

El aumento constante de empresas de transporte, favorecido por el crecimiento de la ciudad y las propuestas insuficientes del gobierno local y nacional para organizar el servicio, contribuyeron a mantener un servicio basado en el interés económico de los empresarios, la necesidad de movilidad de los ciudadanos y las precarias condiciones laborales de los conductores. Tal es el caso del subsidio al transporte que, en vez de contribuir a la calidad del servicio, terminó siendo utilizado por algunos transportadores para percibir mayores recursos económicos.

Aunque se crearon instituciones como el Intra, la Secretaría de Tránsito o Protrans, y se publicaron documentos tendientes a organizar el sector del transporte público, estas iniciativas se quedaron en un nivel diagnóstico, sin propuestas reales de organización y cambios en el servicio de movilidad para la ciudad. Por ello, fueron los empresarios en busca de beneficios económicos y los ciudadanos, con sus necesidades, los que terminaron por trazar los recorridos de los buses, hacía lugares donde en algunas ocasiones aún no existían vías. En esta continua expansión de la ciudad y de los recorridos del transporte público, también fueron los conductores en sus buses partícipes de la configuración de la ciudad y sus dinámicas urbanas. A pesar de las críticas constantes y de la mala calidad del servicio, los transportadores lograron responder a las necesidades de movilidad de los ciudadanos y cumplieron un papel importante en la expansión urbana, al ser facilitadores de la conexión entre los barrios informales y la ciudad formal.

Los problemas que generó el sostenimiento de un único modelo de transporte público colectivo y su operación en manos de sectores privados a lo largo del siglo xx, llevaron a una dependencia de la ciudadanía hacia los empresarios del transporte como garantes de la movilidad de la ciudad, a pesar de ser un servicio que debía ser garantizado por el Estado.

\section{Referencias}

Acevedo, J., Salazar, C. y Castañeda, W. (1993). El metro de Medellín: una ilusión costeada por todos los colombianos. Bogotá: Fonade.

Aprile-Gniset, J. (1992). La ciudad colombiana: siglo XIX y siglo XX. Bogotá: Banco Popular.

Bonilla, R. (2012). Modelos urbanísticos de Cali en el siglo XX: una visión desde la morfología urbana. En G. Loaiza. Historia de Cali siglo XX, t. I: Espacio urbano. Cali: Universidad del Valle. 
Cifuentes, I. (1986). Legislación del transporte terrestre automotor. Normas vigentes. Bogotá: Editorial Presencia.

Jaramillo, S. (1985). El transporte colectivo en Bogotá: los límites de la informalidad. En Transporte y servicios urbanos en América Latina: Actas del Taller de Investigación, t. I. Quito: Inrets.

Mosquera, G. (1984). Morfología, desarrollo y autoconstrucción en Cali: Algunos apartes de la investigación. Cali: Universidad del Valle.

Ortiz, D. y Villarreal, S. (2016). Transporte y mercado interno en Colombia: una contribución a un debate hasta ahora desconocido, 1928-1950. tiempo\&economía, 3(1), 83-107.

Ruíz, A. (2014). Espacio y poblamiento en la ladera sur occidental de Cali: sector Siloé, décadas de 1910 a 2010. Cali: Universidad del Valle.

Vásquez, É. (1980). Ensayos sobre la historia del desarrollo urbano en Cali. Historia y Espacio, 5, $8-63$.

Vásquez, É. (2001). Historia de Cali en el siglo 20: Sociedad, economía, cultura y espacio. Cali: Artes Gráficas del Valle.

Vinasco, D. (2017). Transporte público en Cali: aspectos generales de su configuración en el siglo XX. Revista CS, 21, 41-67.

Documentos institucionales

Centro de administración local integrada, Comuna 14. (2003). Plan de desarrollo estratégico Comuna 15. Cali: Departamento Administrativo de Planeación-Alcaldía de Cali.

Centro de administración local integrada, Comuna 14. (2003). Plan de desarrollo estratégico Comuna 15. Cali: Departamento Administrativo de Planeación-Alcaldía de Cali.

Intra. (1973). Reestructuración rutas urbanas. Cali: Instituto Nacional del transporte-Regional Valle del Cauca.

Oficina de Planeación Municipal de Cali. (1971). Plan general de desarrollo de Cali y su área metropolitana 1970-1985-2000. Cali: Oficina de Planeación municipal-Alcaldía de Cali.

Oficina de Planeación Municipal de Cali. (1980). Plan Integral de desarrollo de Cali y su área de influencia. Documento 17: Situación del transporte. Cali: Alcaldía de Cali.

Secretaría de tránsito y transporte municipal de Cali. (1978). Evaluación transporte público urbano en Cali. Cali: Secretaría de Tránsito y Transporte Municipal-Alcaldía de Cali.

Protrans. (1981). Términos de referencia para una propuesta técnica sobre: Corredores de transporte rápido masivo para Cali y su área inmediata de influencia. Cali: Protrans.

Protrans y Ansaldo Transporti S.P.A. (1983). Estudio de posibles soluciones para el transporte público masivo en el área del Valle geográfico del Río Cauca, t. V, Memorias. Cali: Protrans.

Archivos consultados

Archivo Histórico de Cali. Fondo Alcaldía. 
En línea

Archivo de acuerdos del Concejo municipal de Cali. <http://www.concejodecali.gov.co/Documentos/Acuerdos>

Archivo de decretos Alcaldía de Cali. <ftp://ftp.cali.gov.co/DECRETOS/>

Archivo de decretos Presidencia de Colombia. <ftp://ftp.camara.gov.co/camara/basedoc/decreto/>

Archivo de sentencias de la Corte Suprema de Justicia de Colombia. <ftp://ftp.camara.gov.co/ camara/basedoc/csj_nf/sp/>

Archivo del patrimonio fotográfico y fílmico del Valle del Cauca. <http://bibliotecadigital.icesi. edu.co/biblioteca_digital/handle/10906/5698>

Biblioteca virtual de tratados del Ministerio de relaciones exteriores de Colombia. <http:// apw.cancilleria.gov.co/tratados/SitePages/Menu.aspx>

Centro de documentación Instituto de desarrollo urbano de Bogotá. <http://webidu.idu.gov. co:9090/pmb/opac_css/index.php?lvl=index> 


\section{Anexo}

\begin{tabular}{|c|c|c|c|c|}
\hline \multicolumn{5}{|c|}{$\begin{array}{l}\text { Acciones llevadas a cabo por el gobierno nacional y local por periodos y número de empresas } \\
\text { y rutas de buses }\end{array}$} \\
\hline Actores & Sector & Década de 1970 & Década de 1980 & Inicios década de 1990 \\
\hline \multirow{3}{*}{$\begin{array}{l}\text { Gobierno } \\
\text { Nacional }\end{array}$} & Planeación & $\begin{array}{r}\text { Institucionalizaci } \\
\text { Ilo a nivel nacior } \\
\text { del crecimiento } \\
\text { nentes }\end{array}$ & $\begin{array}{l}\text { n de los planes de desarro- } \\
\text { l regional y local. Además } \\
\text { sico, se incluyeron compo- } \\
\text { ocio-económicos }\end{array}$ & $\begin{array}{l}\text { Nueva reforma a los planes de } \\
\text { desarrollo }\end{array}$ \\
\hline & \multirow[b]{2}{*}{ Transporte } & $\begin{array}{l}\text { Creación del } \\
\text { Intra }\end{array}$ & \multirow{2}{*}{$\begin{array}{l}\text { Creación de Transporte } \\
\text { sin subsidio e inicio de } \\
\text { desmonte progresivo de } \\
\text { subsidios al transporte } \\
\text { público }\end{array}$} & Eliminación del Intra \\
\hline & & $\begin{array}{l}\text { Creación de } \\
\text { subsidio al } \\
\text { transporte }\end{array}$ & & $\begin{array}{l}\text { Desaparición del subsidio al } \\
\text { transporte }\end{array}$ \\
\hline \multirow{3}{*}{$\begin{array}{l}\text { Gobierno } \\
\text { Local }\end{array}$} & \multirow{2}{*}{ Planeación } & $\begin{array}{l}\text { Creación Oficina } \\
\text { de Planeación } \\
\text { Municipal }\end{array}$ & \multirow{2}{*}{$\begin{array}{c}\text { Plan integral de } \\
\text { Desarrollo de Cali (Pideca) }\end{array}$} & \multirow{2}{*}{$\begin{array}{c}\text { Planes de desarrollo a largo } \\
\text { plazo remplazados por planes } \\
\text { correspondientes a cada periodo } \\
\text { de alcaldía }\end{array}$} \\
\hline & & $\begin{array}{l}\text { Plan General de } \\
\text { Desarrollo de } \\
\text { Cali (PGD) }\end{array}$ & & \\
\hline & Transporte & $\begin{array}{l}\text { Creación Secre- } \\
\text { taría de tránsito }\end{array}$ & $\begin{array}{l}\text { Creación de Protrans y } \\
\text { formulación de proyecto } \\
\text { de Tren Metropolitano }\end{array}$ & $\begin{array}{c}\text { El manejo del transporte público } \\
\text { pasa totalmente a manos del } \\
\text { gobierno local }\end{array}$ \\
\hline \multirow{2}{*}{$\begin{array}{l}\text { Empresas de } \\
\text { transporte }\end{array}$} & Número de empresas & 16 & 16 & 23 \\
\hline & $\begin{array}{l}\text { Número de rutas de } \\
\text { buses }\end{array}$ & 33 & 44 & 124 \\
\hline
\end{tabular}

